\title{
Dissipation Induced Nonstationarity in a Quantum Gas
}

\author{
Berislav Buča $\oplus^{1}$ and Dieter Jaksch ${ }^{1,2}$ \\ ${ }^{1}$ Clarendon Laboratory, University of Oxford, Parks Road, Oxford OX1 3PU, United Kingdom \\ ${ }^{2}$ Centre for Quantum Technologies, National University of Singapore, 3 Science Drive 2, Singapore 117543
}

(Received 9 June 2019; published 23 December 2019)

\begin{abstract}
Nonstationary longtime dynamics was recently observed in a driven two-component Bose-Einstein condensate coupled to an optical cavity [N. Dogra, M. Landini, K. Kroeger, L. Hruby, T. Donner, and T. Esslinger, arXiv:1901.05974] and analyzed in mean-field theory. We solve the underlying model in the thermodynamic limit and show that this system is always dynamically unstable — even when mean-field theory predicts stability. Instabilities always occur in higher-order correlation functions leading to squeezing and entanglement induced by cavity dissipation. The dynamics may be understood as the formation of a dissipative time crystal. We use perturbation theory for finite system sizes to confirm the nonstationary behavior.
\end{abstract}

DOI: 10.1103/PhysRevLett.123.260401

Introduction.-Quantum systems composed of many degrees of freedom are expected to relax to stationarity in the longtime limit. This basic principle has been the subject of intense theoretical and experimental research in recent years, in both equilibrium and nonequilibrium settings and for both open and closed systems; see, e.g., Refs. [1,2]. Moreover, relaxation to stationarity was shown to happen on relatively short timescales [3]. In closed systems it is mathematically understood by arguing that every observable evolves as $\langle O(t)\rangle=\sum_{n, m} e^{i \omega_{n m} t} c_{n m}$, where $\omega_{n m}=E_{n}-E_{m}$. The coefficients $c_{n m}$ are determined by the initial state, and $E_{n}$ is the eigenvalue of the Hamiltonian $H$ and eigenstate $\left|E_{n}\right\rangle$. For generic observables and initial states the eigenfrequencies $\omega_{n m}$ entering into the time evolution will be dense and incommensurate. This will lead to mutual dephasing (destructive interference) and relaxation to a time-independent (stationary) value of $\langle O(t)\rangle$ in the longtime limit [4,5].

In contrast, nonstationary dynamics of macroscopic systems is ubiquitous in nature. The emergence of such behavior from the underlying laws of quantum mechanics is an important open question that has far-reaching implications. A possible way for achieving this is for dissipation to dampen all but select equidistantly spaced frequencies $n \omega_{0}$ with $n=1,2,3, \ldots$, thus preventing eigenstate thermalization as discussed in Ref. [6] This is to be contrasted with the emergent stationarity due to the dense incommensurate spectrum described above. This mechanism underlies quantum synchronization [7] and dissipative time crystals [8], and it may induce more complex longtime dynamics [6] and long-range off-diagonal order [9]. This type of time crystalline behavior is qualitatively different from the standard discrete (or Floquet) time crystal, which is a phase of matter with external time-dependent driving that shows persistent oscillations with a period that differs from the one of the external driving field and that may exist both without $[10,11]$ and with [12-15] dissipation. The phenomenon we will be interested in is emergent nonstationarity in model systems without explicit time-dependent external driving (i.e., in a corotating frame where the dynamical equations are not explicitly time dependent), similar to nonstationarity from certain initial states due to many-body scars [16], or the formation of time crystals in the isolated Heisenberg spin chain [17].

Long-term nonstationary dynamics was observed in a recent experiment studying a two-component BoseEinstein condensate (BEC) coherently coupled to two different spatial atomic configurations [18], referred to as the density mode and the spin mode. The coherent couplings are mediated by photons scattered by the atomic system from a transverse pump field into an optical cavity [19]. The experiment showed a rich phase diagram with a dissipation induced region of instability characterized by persistent oscillations. The system was analyzed in meanfield theory, and excellent agreement with the experiment was obtained [19].

In this Letter we provide a solution to the longtime dynamics of the underlying model in the thermodynamic limit by employing the approach pioneered by Emary and Brandes for studying the quantum fluctuations around the mean-field solution [20]. Our main result is that this system displays nonstationary dynamics for all choices of external parameters. In regions where mean-field theory predicts stability, nonstationarity is confined to higher-order correlation functions and counterintuitively leads to dissipation induced squeezing and entanglement which is not present in the corresponding closed system. We confirm our findings using perturbation theory for a finite system.

Two-component BEC coupled to a cavity.-We model the experimental setup studied in Ref. [19] in the Lindblad 
master equation framework [21,22]. By moving to a corotating frame, we eliminate the (simple) time dependence coming from the external high-frequency driving, and our starting point is a time-independent master equation,

$$
\begin{aligned}
\frac{d}{d t} \rho(t) & =\hat{\mathcal{L}} \rho(t) \\
& :=-i[H, \rho(t)]+\kappa\left[2 L \rho(t) L^{\dagger}-\left\{L^{\dagger} L, \rho(t)\right\}\right],
\end{aligned}
$$

taking

$$
\begin{aligned}
H= & \hbar \omega a^{\dagger} a+\hbar \omega_{0}\left(J_{z,+}+J_{z,-}\right) \\
& +\frac{\hbar}{\sqrt{N}}\left[\lambda_{D}\left(a^{\dagger}+a\right)\left(J_{x,+}+J_{x,-}\right)\right. \\
& \left.+i \lambda_{S}\left(a^{\dagger}-a\right)\left(J_{x,+}-J_{x,-}\right)\right],
\end{aligned}
$$

where $a\left(a^{\dagger}\right)$ is the annihilation (creation) operator of the cavity mode, $\omega$ is the detuning between the cavity resonance and the transverse pump field, $J_{\alpha,+}\left(J_{\alpha,-}\right)$ are the collective spin operators of the $+(-)$ Zeeman state separated by angular frequency $\omega_{0}$, and $\lambda_{D, S}$ are the coupling strengths of the atomic spins to the cavity mode. The cavity loss is modeled by a single Lindblad operator $L=a$ with rate $\kappa$.

We extend the approach of Refs. [23,24] to study this system in the thermodynamic limit with the number of particles in the BECs $N \rightarrow \infty$. More specifically, the approach will allow us to study the quantum fluctuations in the leading order of large $N$ around the mean-field solutions. We begin by performing a Holstein-Primakoff transformation,

$$
\begin{aligned}
& J_{+, \pm}=b_{1,2}^{\dagger} \sqrt{N-b_{1,2}^{\dagger} b_{1,2}}, \quad J_{-, \pm}=J_{+, \pm}^{\dagger}, \\
& J_{z, \pm}=b_{1,2}^{\dagger} b_{1,2}-N / 2,
\end{aligned}
$$

where $b_{1}\left(b_{2}\right)$ is the bosonic annihilation operator for the $+(-)$ BEC. Anticipating instabilities of the ground state with $\left\langle J_{z, \pm}\right\rangle=-N / 2$ and $\left\langle J_{+, \pm}\right\rangle=0$ as already obtained in a mean-field treatment [19], we also perform a shift

$$
a \rightarrow a+\alpha \sqrt{N}, \quad b_{1,2} \rightarrow b_{1,2}-\sqrt{\beta_{1,2}} \sqrt{N} .
$$

We expand the Liouvillian in orders of $N$ keeping only powers higher than 0 . The values of $\alpha, \beta_{1,2}$ are then determined by demanding that the resulting Liouvillian is quadratic. We call $\alpha=\beta_{1,2}=0$ the normal case and use superradiant otherwise. Finite values of $\alpha, \beta_{1,2}$ physically correspond to $\left\langle J_{+, \pm}\right\rangle$acquiring a nonzero macroscopic mean-field value. Our Letter does not capture the transient build up of the mean-field values $\alpha, \beta_{1,2}$ and thus describes the longtime dynamics. Note that unphysical solutions to $\alpha$, $\beta_{1,2}$ must be discarded by hand.

In the normal case we obtain for the Hamiltonian (up to an irrelevant constant shift)

$$
\begin{aligned}
H= & \omega a^{\dagger} a+\omega_{0}\left(b_{1}^{\dagger} b_{1}+b_{2}^{\dagger} b_{2}\right) \\
& +\lambda_{D}\left(a+a^{\dagger}\right)\left(b_{1}+b_{1}^{\dagger}+b_{2}+b_{2}^{\dagger}\right) \\
& +i \lambda_{S}\left(a-a^{\dagger}\right)\left(b_{1}+b_{1}^{\dagger}-b_{2}-b_{2}^{\dagger}\right) .
\end{aligned}
$$

In the superradiant case the Hamiltonian also contains squeezing terms like $b_{1,2}^{2}$ and $\left(b_{1,2}^{\dagger}\right)^{2}$, and it is given in the Supplemental Material [25]. The Lindblad operators remain the same following a shift that removes the linear terms in $\hat{\mathcal{L}}$.

Since the resulting Liouvillian is quadratic, we solve it exactly using the method of "third quantization" [26,27]. The details are in the Supplemental Material [25]. Expanding the eigenvalues for large $\kappa$, we get in the first two leading orders

$$
\lambda_{n_{1}, n_{2}}=i n_{1} \omega_{0}+\frac{2 n_{2}}{\kappa} \Gamma^{2}+\mathrm{O}\left(\frac{1}{\kappa^{2}}\right),
$$

where now $n_{1,2}=0, \pm 1, \pm 2, \ldots$. In the normal case $\Gamma^{2}=V^{2} \equiv\left(\lambda_{D}^{2}+\lambda_{S}^{2}\right)$, and it can be found numerically in the superradiant case.

Eigenvalues with a positive real part are an unphysical consequence of the unbounded bosonic Liouvillian superoperator. The eigenmodes corresponding to these eigenvalues "blow up" and signal an instability. This instability arises from the coupling of the lossy cavity to the BECs. We emphasize that the leading imaginary parts of the eigenvalues are equally spaced, and thus dephasing of the dynamics is prevented $[4,5]$. This is in contrast to the related closed Dicke model, which possesses a dense spectrum and is known to exhibit chaos and thermalization [20] (see the Supplemental Material [25] for more details). It demonstrates how dissipative engineering of the spectrum by coupling to the lossy cavity prevents thermalization.

Our results are consistent with those obtained in meanfield theory in Ref. [19]. Fluctuations around the initial BEC state will be amplified on a timescale given by $\kappa / \Gamma^{2}$. The system will dynamically evolve away from the initial state and may show persistent oscillations with frequency $\approx \omega_{0}$. In order to analyze how this instability manifests itself in the dynamics of observables, we now move on to the Heisenberg picture.

Equations of motions.-The quadratic Liouvillian admits a finite closed set of Heisenberg equations of motion for $\vec{a}=\left(a, b_{1}, b_{2}, a^{\dagger}, b_{1}^{\dagger}, b_{2}^{\dagger}\right)$ of the form $\dot{\vec{a}}=\hat{\mathcal{L}}^{\dagger} \vec{a}$. It preserves the Gaussian nature of quantum states, and hence the system dynamics is fully determined by the one- and twopoint observables. For the one-point functions in the normal case, the expectation values evolve according to

$$
\begin{aligned}
\langle\dot{a}(t)\rangle= & -\frac{i \lambda_{D}\left[q_{1}^{*}(t)+q_{1}(t)\right]}{\sqrt{N}}+\frac{\lambda_{S}\left[q_{2}(t)^{*}+q_{2}(t)\right]}{\sqrt{N}} \\
& -\kappa\langle a(t)\rangle-i \omega\langle a(t)\rangle, \\
\left\langle\dot{b_{j}}(t)\right\rangle= & -\frac{i \lambda_{D} q_{0}(t)}{\sqrt{N}}+(-1)^{j-1} \frac{\lambda_{S} q_{0}^{\prime}(t)}{\sqrt{N}}-i \omega_{0}\left\langle b_{j}(t)\right\rangle,
\end{aligned}
$$


where $q_{0}(t)=\left\langle a^{\dagger}(t)\right\rangle+\langle a(t)\rangle, q_{0}^{\prime}(t)=\left\langle a^{\dagger}(t)\right\rangle-\langle a(t)\rangle$, $q_{1}(t)=\left\langle b_{1}(t)\right\rangle+\left\langle b_{2}(t)\right\rangle, q_{2}(t)=\left\langle b_{1}(t)\right\rangle-\left\langle b_{2}(t)\right\rangle$. After adiabatic elimination of the cavity mode, we obtain agreement with the mean-field treatment in Ref. [19]. The superradiant case is treated analogously [25].

We perform a stability analysis of one- and two-point correlators in Fig. 1 showing the maximum rate at which a fluctuation around the stationary solutions determined by $\alpha$ and $\beta_{1,2}$ can be exponentially amplified. The normal case shown in Fig. 1(a) exhibits only a small region around $\phi=$ $\arctan \left(\lambda_{S} / \lambda_{D}\right)$ where the one-point correlators are stable. This region increases with decreasing strength of the cavity coupling $V$. The instability of the one-point function is accompanied by oscillations with a frequency around $\omega_{0}$ shown in Fig. 1(b) almost everywhere. In contrast, the superradiant case shown in Fig. 1(c) is stable for most values of $\phi$ and $\omega$ for the chosen parameters. In Fig. 1(d) we see that there is a small part of the phase diagram where neither the normal nor any of the superradiant solutions are stable in the one-point functions. We find that the region of instability around $\phi=90^{\circ}$ and $\phi=0$ increases with decreasing $V$.

The stability analysis for the two-point correlators gives a different picture. They are also unstable for all of the superradiant solutions except in the points where the model reduces to the Dicke model $\left(\lambda_{D}=0\right.$ or $\left.\lambda_{S}=0\right)$. This is consistent with some of the eigenvalues of the Liouvillian obtained from the rapidities having a positive real part, which implies that some of the observables must be unstable.

(a)
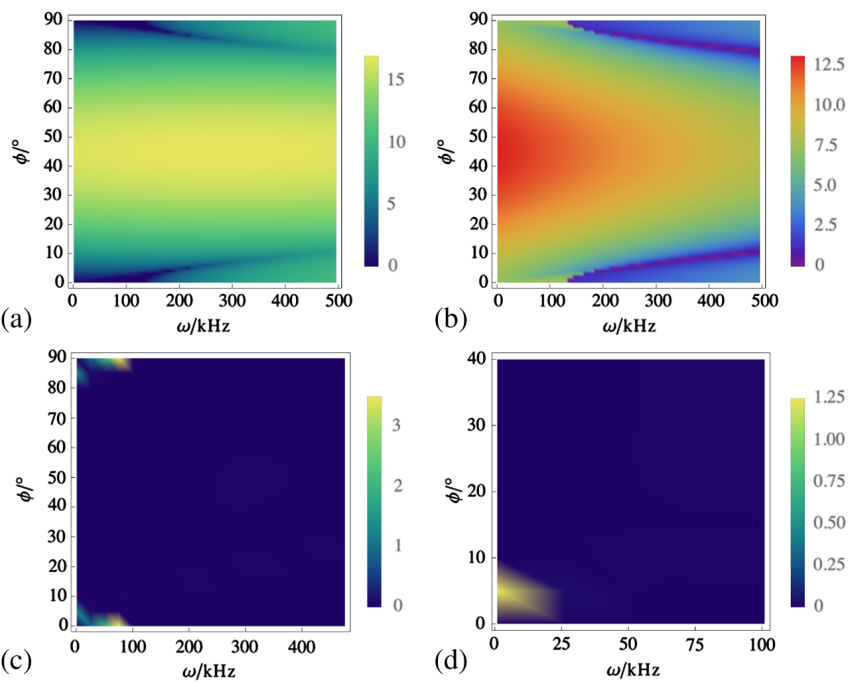

FIG. 1. Stability analysis of the Holstein-Primakoff solutions giving the maximum real part of the eigenvalues' time evolution generator for (a) the one-point functions in the normal case, and (c) the most stable of the superradiant solutions. (d) A nontrivial part of the phase diagram enlargement indicating the maximum real part of the most stable of either the normal or the superradiant case. The imaginary part (giving the frequencies of the oscillations) corresponding to (a) is shown in (b). We use the parametrization $\lambda_{D}=V \cos \phi, \lambda_{S}=V \sin \phi$ with $V=121.65 \mathrm{kHz}$ and $\omega_{0}=$ $7.4 \mathrm{kHz}, \kappa=1.25 \mathrm{MHz}$.
The instabilities are observable in connected twopoint correlation functions of the form $\left\langle X_{1} X_{2}\right\rangle_{c}:=$ $\left\langle X_{1} X_{2}\right\rangle-\left\langle X_{1}\right\rangle\left\langle X_{2}\right\rangle$, where $X_{1,2}$ are BEC observables, and we show examples in Fig. 2. These are related to spin squeezing [28,29] (see the Supplemental Material [25]). We find that the spin squeezing parameter in the $y$ direction is oscillatory and can be made arbitrarily small with a suitable initial choice of $\left\langle a^{2}(0)\right\rangle$ [25], indicating entanglement. The nontrivial behavior of the connected correlation function is a clear indication of beyond mean-field behavior which is of purely quantum origin. The system thus exhibits entanglement induced by dissipation, which could also have ramifications on quantum information processing applications.

We see in Fig. 3 that even in the phase where the onepoint functions relax to stationarity, the cross-correlation $\left\langle J_{x,+} J_{x,-}\right\rangle_{c}$ and $\left\langle J_{x,+} J_{y,-}\right\rangle_{c}$ functions are nonstationary.

Thus the apparent contradiction between mean-field theory showing a phase transition from stable to unstable normal solutions and a full quantum treatment always giving nondecaying eigenvalues with a finite imaginary part is resolved: the phase transition takes place for one-point correlators only, while higher-order correlations are always unstable. Since there exist eigenmodes of the quantum Liouvillian with eigenvalues that have $\operatorname{Re}(\lambda) \geq 0$, $\operatorname{Im}(\lambda) \neq 0$, there will always be some observables for some initial condition that will persistently oscillate.

Perturbation theory for finite system size.-The positive real eigenvalues of the Liouvillian in the thermodynamic limit are unphysical and an artifact of the unboundedness of the Liouvillian. Furthermore, since we kept only the linearized quantum fluctuations in the Holstein-Primakoff expansion, it is possible that higher-order correlators qualitatively modify the main results above. To address these issues, we study the system using perturbation theory. We find that the short-time dynamics of a system of any size are
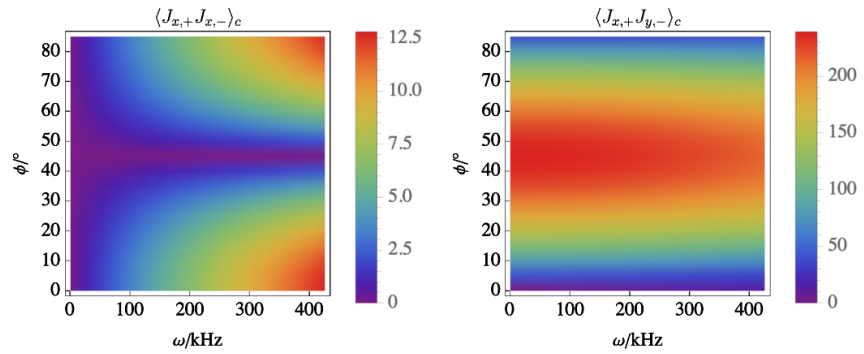

FIG. 2. Exemplary maximum values of two-point functions calculated by time evolving the equations of motions for up to $t=1 \mathrm{~ms}$ from the ground state of the two BECs. We plot the amplitude of the oscillations (for the cross-correlators $\left|\left\langle J_{x,+} J_{x,-}\right\rangle_{c}\right|$ and $\left.\left|\left\langle J_{x,+} J_{y,-}\right\rangle_{c}\right|\right)$. We use the parametrization $\lambda_{D}=V \cos \phi, \lambda_{S}=$ $V \sin \phi$ with $V=92.5 \mathrm{kHz}$ and $\omega_{0}=7.4 \mathrm{kHz}, \kappa=1.25 \mathrm{MHz}$. We see clear deviation from the mean-field results. Importantly, even deep inside the phase diagram where there are no oscillations of the mean fields [19], the cross-correlators $\left|\left\langle J_{x,+} J_{x,-}\right\rangle_{c}\right|$ and $\left|\left\langle J_{x,+} J_{y,-}\right\rangle_{c}\right|$ do show oscillating behavior. 

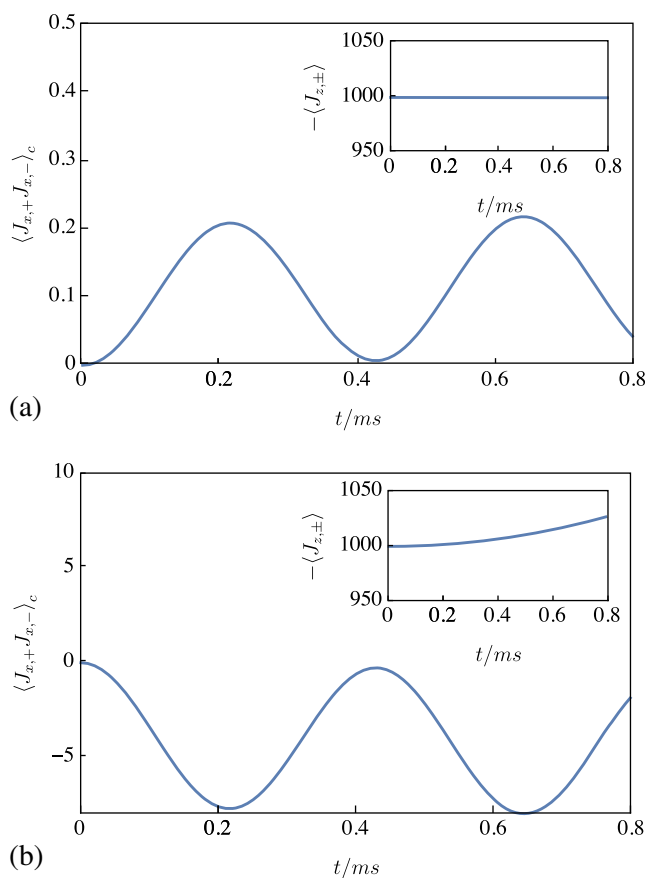

FIG. 3. The cross-correlation functions $\left\langle J_{x,+} J_{x,-}\right\rangle_{c}=$ $\left\langle J_{x,+} J_{x,-}\right\rangle-\left\langle J_{x,+}\right\rangle\left\langle J_{x,-}\right\rangle$ (a) inside the phase where the onepoint functions $\left\langle J_{x, \pm}\right\rangle$ oscillate, $\lambda_{D}=6.3 \mathrm{kHz}, \lambda_{S}=7.25 \mathrm{kHz}$, $\omega=46 \mathrm{kHz}$, (b) and in the phase where they are stationary, $\lambda_{D}=9.6 \mathrm{kHz}, \lambda_{S}=0.17 \mathrm{kHz}, \omega=246 \mathrm{kHz}$. The frequency, atom number, and cavity loss are $\omega_{0}=7.4 \mathrm{kHz}, N=2000$, and $\kappa=1.25 \mathrm{MHz}$ in both cases. The insets show that $\left\langle J_{z, \pm}\right\rangle \approx-N / 2$, thus validating the assumptions of Ref. [19].

well described by the results of the Holstein-Primakoff approach [25]. More specifically, we obtain eigenfrequencies that are integer multiples of $\omega_{0}$ at leading order for the longest lived oscillations. The corresponding eigenmodes are given in the Supplemental Material [25] and are perturbatively close to the vacuum state of the cavity.

Using simple large deviation arguments [30], it is trivial to argue that the number of photons leaving the cavity should also be low. This is consistent with experimental results in the dynamical instability region [Fig. 2(a) of Ref. [19] ]. Thus we recover the eigenfrequencies of the persistent oscillations, but without the unphysical unbounded increase in the expectation values.

Conclusion.-By taking the thermodynamic limit, we exactly solved a model of a driven two-component BoseEinstein condensate coupled to an optical cavity undergoing dissipation [19]. We identified that the system has both normal and superradiant behavior (in which the modes acquire macroscopic mean values) [20,23]. We find longtime oscillations in the model due to the existence of eigenvalues with non-negative real and imaginary parts which are close to integer multiples of $i \omega_{0}$. The imaginary part corresponds to the frequencies of the persistent oscillations of observables. The fact that these frequencies are almost equidistant means that eigenstate dephasing $[4,5]$ of the dynamics is impossible. In contrast, if the cavity were closed, we would have densely spaced and incommensurate frequencies in the Hamiltonian that could mutually dephase and lead to equilibration like in the related Dicke model [31,32]. (See also Refs. [33,34] for a related semiclassical treatment of equilibration.)

We thus conclude that we have an example of dissipation induced dynamics in a system that would otherwise equilibrate, akin to a dissipative time crystal [8]. The system in the laboratory frame is time dependent due to the (simple) high-frequency driving [19]. Moving to the corotating frame leaves us with a time-independent master equation (1). The persistent oscillations arise in the rotating frame from the interplay between the external drive inputing energy into the system and the cooling from the cavity loss. Without the driving the cavity would simply be empty, and without the dissipation the system would heat and thermalize. A sketch of such a mechanism as a possibility for realizing discrete time crystals was proposed in Ref. [35], but here we have identified an actual physical system with this property. The system we study is better understood in the corotating frame as an example of continuous time symmetry breaking induced by dissipation as the period of the oscillatory response in the laboratory frame bears no fixed relation to the driving period. In other words, the system does not realize a discrete-time crystalline structure in the laboratory frame.

We discuss evidence of spin squeezing in the Supplemental Material [25] which should imply entanglement [29]. We validate the thermodynamic results by taking the large cavity limit and performing perturbation theory for finite systems. We find in the leading order of this perturbation the dark Hamiltonian criteria of Ref. [6] are trivially satisfied. The persistent oscillations may thus also be understood as an example of quantum Zeno dynamics (see, e.g., Refs. [36-38]).

In addition to the persistent oscillations at frequencies close to $\omega_{0}$, the dynamics also entangles the condensates, leading to strong squeezing through the dissipative coupling. These features are not captured by mean-field theory, which instead assumes the condensates to be decoupled.

In the future we plan to investigate the implications of dissipation induced nonstationary squeezing and entanglement, e.g., for quantum enhanced metrology [39].

We thank N. Dogra and T. Esslinger both for sharing their preliminary results with us and for the useful discussion. We also thank A. Dietrich and C. Sanchez Munoz for the useful discussions. The work here was supported by EPSRC Programme Grant No. EP/P009565/1 and the European Research Council under the European Unions Seventh Framework Programme (FP7/2007-2013)/ERC Grant Agreement No. 319286 Q-MAC.

Note added.-Recently, Ref. [40] appeared, which discusses extensions of the mean-field results of Ref. [19]. We go beyond both and study the quantum model. 
[1] D. Rossini, A. Silva, G. Mussardo, and G. E. Santoro, Phys. Rev. Lett. 102, 127204 (2009); P. Calabrese, F. H. L. Essler, and M. Fagotti, Phys. Rev. Lett. 106, 227203 (2011); J.-S. Caux and R. M. Konik, Phys. Rev. Lett. 109, 175301 (2012); P. Barmettler, M. Punk, V. Gritsev, E. Demler, and E. Altman, Phys. Rev. Lett. 102, 130603 (2009); M. Collura, S. Sotiriadis, and P. Calabrese, Phys. Rev. Lett. 110, 245301 (2013).

[2] O. A. Castro-Alvaredo, B. Doyon, and T. Yoshimura, Phys. Rev. X 6, 041065 (2016); B. Bertini, M. Collura, J. De Nardis, and M. Fagotti, Phys. Rev. Lett. 117, 207201 (2016); M. Ljubotina, M. Žnidarič, and T. Prosen, Nat. Commun. 8, 16117 (2017); J. J. Mendoza-Arenas, S. Al-Assam, S. R. Clark, and D. Jaksch, J. Stat. Mech. (2013) P07007; J. J. Mendoza-Arenas, T. Grujic, D. Jaksch, and S. R. Clark, Phys. Rev. B 87, 235130 (2013).

[3] L. D'Alessio, Y. Kafri, A. Polkovnikov, and M. Rigol, Adv. Phys. 65, 239 (2016).

[4] T. Barthel and U. Schollwöck, Phys. Rev. Lett. 100, 100601 (2008).

[5] F. H. L. Essler and M. Fagotti, J. Stat. Mech. (2016) 064002.

[6] B. Buca, J. Tindall, and D. Jaksch, Nat. Commun. 10, 1730 (2019).

[7] C. Davis-Tilley and A. D. Armour, Phys. Rev. A 94, 063819 (2016); T. E. Lee and H. R. Sadeghpour, Phys. Rev. Lett. 111, 234101 (2013); N. Lörch, S. E. Nigg, A. Nunnenkamp, R. P. Tiwari, and C. Bruder, Phys. Rev. Lett. 118, 243602 (2017); N. Jaseem, M. Hajdušek, V. Vedral, R. Fazio, L.-C. Kwek, and S. Vinjanampathy, arXiv:1812.10082; A. Roulet and C. Bruder, Phys. Rev. Lett. 121, 063601 (2018); 121, 053601 (2018); B. Bellomo, G. L. Giorgi, G. M. Palma, and R. Zambrini, Phys. Rev. A 95, 043807 (2017); M. Xu, D. A. Tieri, E. C. Fine, J. K. Thompson, and M. J. Holland, Phys. Rev. Lett. 113, 154101 (2014); J. Tindall, C. Sanchez Munoz, B. Buca, and D. Jaksch, arXiv:1907.12837.

[8] C.-K. Chan, T. E. Lee, and S. Gopalakrishnan, Phys. Rev. A 91, 051601(R) (2015); F. Iemini, A. Russomanno, J. Keeling, M. Schiró, M. Dalmonte, and R. Fazio, Phys. Rev. Lett. 121, 035301 (2018); K. Tucker, B. Zhu, R. J. Lewis-Swan, J. Marino, F. Jimenez, J. G. Restrepo, and A. M. Rey, New J. Phys. 20, 123003 (2018); D. Barberena, R. J. Lewis-Swan, J. K. Thompson, and A. M. Rey, Phys. Rev. A 99, 053411 (2019); C. Lledó, Th. K. Mavrogordatos, and M. H. Szymańska, Phys. Rev. B 100, 054303 (2019); B. Zhu, J. Marino, N. Y. Yao, M. D. Lukin, and E. A. Demler, New J. Phys. 21, 073028 (2019); H. Kessler, J. G. Cosme, M. Hemmerling, L. Mathey, and A. Hemmerich, Phys. Rev. A 99, 053605 (2019); K. Seibold, R. Rota, and V. Savona, arXiv:1910.03499.

[9] J. Tindall, B. Buca, J. R. Coulthard, and D. Jaksch, Phys. Rev. Lett. 123, 030603 (2019).

[10] D. V. Else, B. Bauer, and C. Nayak, Phys. Rev. Lett. 117, 090402 (2016).

[11] A. Lazarides, A. Das, and R. Moessner, Phys. Rev. Lett. 112, 150401 (2014).

[12] F. M. Gambetta, F. Carollo, A. Lazarides, I. Lesanovsky, and J. P. Garrahan, arXiv:1905.08826.
[13] F. M. Gambetta, F. Carollo, M. Marcuzzi, J. P. Garrahan, and I. Lesanovsky, Phys. Rev. Lett. 122, 015701 (2019).

[14] A. Lazarides, S. Roy, F. Piazza, and R. Moessner, arXiv: 1904.04820 .

[15] Z. Gong, R. Hamazaki, and M. Ueda, Phys. Rev. Lett. 120, 040404 (2018).

[16] C. J. Turner, A. A. Michailidis, D. A. Abanin, M. Serbyn, and Z. Papic, Nat. Phys. 14, 745 (2018).

[17] M. Medenjak, B. Buca, and D. Jaksch, arXiv:1905.08266.

[18] M. Landini, N. Dogra, K. Kroeger, L. Hruby, T. Donner, and T. Esslinger, Phys. Rev. Lett. 120, 223602 (2018).

[19] N. Dogra, M. Landini, K. Kroeger, L. Hruby, T. Donner, and T. Esslinger, arXiv:1901.05974.

[20] C. Emary and T. Brandes, Phys. Rev. E 67, 066203 (2003).

[21] H.-P. Breuer and F. Petruccione, The Theory of Open Quantum Systems (Oxford University Press, New York, 2002).

[22] G. Lindblad, Commun. Math. Phys. 48, 119 (1976).

[23] F. Dimer, B. Estienne, A. S. Parkins, and H. J. Carmichael, Phys. Rev. A 75, 013804 (2007).

[24] E. M. Kessler, G. Giedke, A. Imamoglu, S. F. Yelin, M. D. Lukin, and J. I. Cirac, Phys. Rev. A 86, 012116 (2012).

[25] See Supplemental Material at http://link.aps.org/supplemental/ 10.1103/PhysRevLett.123.260401 for details of the HolsteinPrimakoff and Bogoliubov transformations, third quantization procedure, perturbation theory and a study of the closed system and semiclassical limit.

[26] T. Prosen, New J. Phys. 10, 043026 (2008).

[27] T. Prosen and T.H. Seligman, J. Phys. A 43, 392004 (2010).

[28] M. Kitagawa and M. Ueda, Phys. Rev. A 47, 5138 (1993).

[29] A. Sorensen, L.-M. Duan, I. Cirac, and P. Zoller, Nature (London) 409, 63 (2001).

[30] H Touchette, Phys. Rep. 478, 1 (2009).

[31] A. Altland and F. Haake, Phys. Rev. Lett. 108, 073601 (2012).

[32] A. Altland and F. Haake, New J. Phys. 14, 073011 (2012).

[33] M. Gutzwiller, Chaos in Classical and Quantum Mechanics (Springer, New York, 1990); F. Haake, Quantum Signatures of Chaos (Springer, New York, 2010).

[34] I. Garcia-Mata, A. R. R. Carvalho, F. Mintert, and A. Buchleitner, Phys. Rev. Lett. 98, 120504 (2007); A. Buchleitner, D. Delande, and J. Zakrzewski, Phys. Rep. 368, 409 (2002).

[35] D. V. Else, B. Bauer, and C. Nayak, Phys. Rev. X 7, 011026 (2017).

[36] P. Facchi and S. Pascazio, J. Phys. A 41, 493001 (2008).

[37] K. Macieszczak, M. Guţă, I. Lesanovsky, and J. P. Garrahan, Phys. Rev. Lett. 116, 240404 (2016).

[38] P. Zanardi and L. Campos Venuti, Phys. Rev. Lett. 113, 240406 (2014).

[39] C. S. Munoz, B. Buca, J. Tindall, A. González-Tudela, D. Jaksch, and D. Porras, arXiv:1903.05080.

[40] E. I. Rodríguez Chiacchio and A. Nunnenkamp, Phys. Rev. Lett. 122, 193605 (2019). 